\title{
Editorial: Der Blutige Ernst - Krise und Politik
}

I.

„Der Blutige Ernst“, so hieß eine von George Grosz und Carl Einstein im Jahr 1919 herausgegebene satirische Zeitschrift, die einen schonungslosen Blick auf die gesellschaftlichen Verhältnisse warf. Mit (nicht nur) satirischen Mitteln stellten sie den „blutigen Ernst“ der Lage der bürgerlichen Schönfärberei gegenüber. Um den blutigen Ernst der Krise soll es auch in diesem Heft der PROKLA gehen. Doch ist neuerdings in den Medien zu hören, die Krise - sie heißt jetzt wieder „Rezession“ sei „Schnee von gestern“. Der schlimmste wirtschaftliche Einbruch seit der Nachkriegszeit sei endlich vorüber, die staatliche Systemrettungsmission erfüllt. So stellt der IWF in seinem jüngsten World Economic Outlook fest, dass die Mehrzahl der wichtigen Industriestaaten die wirtschaftliche Talsohle bereits verlassen habe und im Jahr 2010 eine Besserung zu erwarten sei. In Ostasien brummt die Konjunktur tatsächlich schon wieder: Die chinesische Wirtschaft ist bereits in diesem Jahr zu ihrem stürmischen Wachstum von über 8\% zurückgekehrt. Aber auch Deutschland habe das Schlimmste hinter sich. Aktuelle Prognosen betonen, dass im kommenden Jahr ein leichtes Wachstum von 1,2\% zu erwarten sei. Dieser Umschwung sei das Ergebnis der koordinierten Rettungsaktionen der Staats- und Regierungschefs. Wir erinnern uns: Als Reaktion auf die Krise wurden weltweit Billionen an USDollar in die „Bail Outs“ von Banken und in Konjunkturpakete gesteckt. Dabei wurden kurzfristig überkommene wirtschaftspolitische Leitsätze über Bord geworfen: Galt staatliche Politik im neoliberalen Diskurs als unfähig (da der Markt alles besser könne) und ohnmächtig (angesichts der Globalisierung bleibe den Nationalstaaten sowieso keine Eingriffsmöglichkeit mehr), so wurde nun von allen Seiten nach dem Staat als Retter der kapitalistischen Ökonomie gerufen.

Trotz dieser Staatsaktionen hat die Krise jedoch zu katastrophalen Folgen geführt. Der Zusammenbruch auf den Finanzmärkten habe rund 10,5 Billionen US\$ gekostet, rechnet eine neue CommerzbankStudie vor. Werden die Bankenabschreibungen abgezogen, bleiben immer noch fast 4,7 Billionen US\$ an Verlusten auf den Immobilienmärkten und die Kosten des Wirtschaftseinbruchs von rund 4,2 Billionen US\$. Der Welthandel ist im Jahr 2009 um rund 12\% geschrumpft. Die UNOrganisation für Ernährung und Landwirtschaft (FAO) stellt fest, dass im Juni 2009 zum ersten Mal die Marke von einer Milliarde hungernder Menschen überschritten wurde. Dies waren fast 40 Millionen Menschen mehr als im letzten Jahr eine Zunahme, die der Bevölkerung Polens entspricht. Allein in den OECD-Staaten haben seit Ende 2007 über 15 Millionen Menschen - alle Niederländer/innen gewissermaßen - ihren Job verloren, und die Arbeitslosigkeit soll, wenn überhaupt, erst Ende 2010 ihren Höchststand erreichen. Ein Ende der Krise sieht anders aus; für viele Länder hat der blutige Ernst der Krise gerade erst begonnen. In welch unterschiedlicher Weise sich die Krise in den verschiedenen EU-Ländern ausgewirkt hat, wird in dem Beitrag von Joachim Becker und Johannes Jäger aufgezeigt.

Wie wird die Krisengeschichte der Gegenwart weitergehen? Immer wieder wurden in den vergangenen Monaten Erinnerungen an die Große Depression 1929ff geweckt, in der sich Phasen eines kurzen Aufschwungs mit immer tieferen Einbrüchen abwechselten. „Vermeintliche Finanzexperten " riefen damals, so erinnert uns Der Spiegel, ,immer wieder das Ende des Abschwungs aus - und lagen auf fatale Weise daneben." Und noch im Frühling 
2009 waren die Konjunkturverläufe der beiden historischen Einbrüche verblüffend ähnlich, mit dem einzigen Unterschied, dass der Zusammenbruch ab Ende 2008 noch rascher und tiefer war. Da fragt sich nicht nur der Spiegel: „Wiederholt sich die Geschichte doch?"“

Die kritische Sozialgeschichtsschreibung stellt sich dieselbe Frage. Eric Hobsbawm, der große Historiker des 20. Jahrhunderts, fühlt sich durchaus nicht nur wirtschaftsstatistisch an die Weltwirtschaftskrise erinnert. Die milliardenschweren Konjunkturmaßnahmen oder die eilig einberufenen G20-Gipfel stellten eher unbeholfen-kurzfristige Maßnahmen dar, die nur Symptome, nicht aber die Ursachen der Krise bekämpften, so seine These. Und deswegen sei die Situation - „obwohl sich Geschichte nicht wiederholt“ - heute ,ähnlich dramatisch wie damals, nein schlimmer: Keine Regierung weiß, was sie tun soll." Hobsbawm selbst hat in Das Zeitalter der Extreme überzeugend dargestellt, wie der wirtschaftliche Zusammenbruch in den späten 1920er Jahren entscheidend zum „Untergang des Liberalismus“ beitrug. Die bis dahin unvorstellbar hohen Arbeitslosenzahlen, die z.B. 1932-33 in Deutschland zeitweilig Werte von bis zu $44 \%$ erreichten, polarisierten die Gesellschaft in einer Weise, dass fortan nur noch drei große Optionen um die politischintellektuelle Vorherrschaft wetteiferten: Der sowjetmarxistische Kommunismus, ein staatlich reformierter Kapitalismus und der autoritäre Faschismus, der schließlich in seiner deutschen und japanischen Variante - den Zweiten Weltkrieg hervorrief.

Doch Hobsbawm selbst gibt in seinem Buch auch Hinweise auf die Unterschiede zwischen beiden Epochen. In den meisten Staaten Europas kam damals weitaus weniger als ein Viertel der Arbeiterschaft in den Genuss von wirksamer Arbeitslosenunterstützung, während heute selbst in den meisten Schwellenländern Sozialsysteme existieren, die die Krisenfolgen zumindest einigermaßen auffangen. Heute trotzt keine planwirtschaftlich organisierte Sowjetunion als Systemkonkurrent der Krise, sondern mit China ausgerechnet ein Land, das in den vergangenen Jahrzehnten dem Kapitalismus Tür und Tor geöffnet hat. Der damaligen Hoffnung einer Inter- nationale der Arbeiter steht nun eine Internationale der Kapitalisten und Staatschefs gegenüber, die „aufgeschreckt wie Krankenschwestern ans Bett des Kapitalismus" eilt, um irgendetwas zu tun, die aber damit zumindest Eingriffswillen und Handlungsfähigkeit demonstriert hat.

Um den Vergleich der Krise von 1929 mit der gegenwärtigen Krise, geht es auch in dem Beitrag von Ingo Schmidt: Während die Krise von 1929 den endgültigen Aufstieg der USA zur Hegemonialmacht markiert habe, zeige die gegenwärtige Krise den Niedergang dieser Hegemonie.

II.

Es wäre durchaus weiterer Überlegungen wert, inwieweit die historische Diagnostik der Weltwirtschaftskrise, die Hobsbwam in den Befunden „Untergang des Liberalismus“" und „Polarisierung der Gesellschaft“ bündelte, sich auch auf die gegenwärtige (Nach-?)Krisenkonstellation übertragen lässt. Ein der Vorgeschichte des Zweiten Weltkriegs vergleichbarer „Untergang des Liberalismus" ist derzeit jedenfalls noch nicht $\mathrm{zu}$ erkennen. Die neoliberalen Ökonomen à la Hans-Werner Sinn, die für eine kurze (glückliche) Zeit von der Bühne multimedialer Wahrheitsverkündigung abgetreten waren, sind mittlerweile zurückgekehrt und schreiben dicke Bücher darüber, „Wie es zur Finanzkrise kam, und was jetzt zu tun ist" (wir ahnen es schon: zu viel Staat vorher, jetzt weniger Staat vielleicht?). Und bei den Bundestagswahlen ist die Partei des unbeirrbaren Wirtschaftsliberalismus von potenziellen Krisenverlierern aus den abhängig beschäftigten Mittelklassen und den erwartbaren Krisengewinnlern gemeinsam zum größten Wahlerfolg ihrer Geschichte befördert worden. Die Krise hat anscheinend noch nicht auf das Bewusstsein der Mehrheit der Bevölkerung durchgeschlagen. Warum das so ist, diskutieren Klaus Dörre, Michael Behr, Dennis Eversberg und Karen Schierhorn in ihrem auf empirischen Studien beruhenden Aufsatz.

Anderswo hat die Krise allerdings durchaus radikaleren, offen anti-liberalen politischen Strömungen Auftrieb verliehen. In Ungarn beispielsweise gewannen mit der Jobbik und in Rumänien mit der Großrumänienpartei rechte Bewegungen vor dem Hintergrund der Krisengeschehnisse 
an Gewicht und erreichten bei den Europawahlen 2009 erschreckend gute Ergebnisse. Inwiefern die extrem Rechte von der Krise profitiert oder auch nicht, untersucht Christina Kaindl in ihrem Beitrag.

In mehreren Ländern, in denen soziale Bewegungen und Gewerkschaften entschieden gegen die Krisenauswirkungen mobilisierten, ging die Linke gestärkt aus den jüngsten Urnengängen hervor. In Portugal gewann die radikale Linke über $21 \%$ der Stimmen, in Island stellte erstmals eine rot-grüne Linksregierung die Mehrheit.

Auch Deutschland wird Zeuge einer zunehmenden wahlpolitischen Polarisierung. Bei der Bundestagwahl erhielten die Volksparteien CDU/CSU und SPD zusammen nur noch 57\% der Stimmen. Ähnlich wie in Großbritannien Labour ist die SPD mit 23\% auf ihrem historischen Tiefstand angelangt. Seit dem Jahr 1998 hat die Partei 10,2 Millionen Wähler/innen verloren und so ihre soziale Basis halbiert. Die drei „kleinen“ Parteien FDP, Die Linke und Grüne erreichten jeweils ein zweistelliges Ergebnis. Die Wahlbeteiligung war mit $71 \%$ die niedrigste seit der Gründung der Bundesrepublik. Die mit beinahe 15\% der Stimmen große Gewinnerin FDP, die nun eine konservativ-liberale Mehrheit in Bundestag wie Bundesrat möglich macht, konnte wohl davon profitieren, dass sie als einzige Partei der Mitte keine programmatische Erneuerung vor dem Hintergrund der Krise durchsetzen musste und sich als Gralshüterin liberaler Grundsätze präsentieren konnte. Doch auch „Die Linke“ hat mit 12\% ein Ergebnis erreicht, das noch vor wenigen Jahren als unmöglich gegolten hätte und die gesellschaftliche Stärkung antineoliberaler Positionen ausdrückt. Das Ergebnis ist nun eine schwarz-gelbe Koalition, in der mindestens die fünf FDPMinister/innen dafür sorgen werden, dass der Neoliberalismus nicht nur fortgesetzt, sondern sogar vertieft wird. Pläne für den Um- und Abbau des öffentlichen Gesundheitssystems, für einen unsozialen Stufensteuersatz, für die Ablehnung von Mindestlöhnen und für längere Laufzeiten für Atomkraftwerke bedienen so offensichtlich die Interessen von Kapital und oberen Mittelschichten, dass selbst die Wirtschaftsredaktion der $F A Z$ sich überrascht zeigte ob dermaßen unverhohlener bürgerlicher
Klientelpolitik. Das große Wahlversprechen von Steuersenkungen wird - und muss mit Sozialabbau und Staatsschulden erkauft werden. Jüngste Meldungen, dass die Krise in Deutschland erst jetzt auf dem Arbeitsmarkt ankomme und im Jahr 2010 rund 800.000 zusätzliche Arbeitslose zu erwarten seien, werden souverän ignoriert bzw. schon vorsorglich der Vorgängerregierung und ihren sozialdemokratischen Wohltatsministern angekreidet.

Vieles spricht somit dafür, dass die sozialen Folgen der Krise in Deutschland erst zeitlich verschoben richtig zutage treten wenn die viel beschworene „wirtschaftliche Talsohle" bereits durchschritten ist und die Rechnungen für Bankenrettungsmilliarden und symbolische Abwrackprämienpolitik präsentiert werden. Diejenigen, die zahlen müssten, aber alles tun werden, um nicht zu zahlen, wurden durch die Wahlen gestärkt. Hobsbawms zweite historische Diagnose, die der sozialen Polarisierung, ist für die Gegenwart also keineswegs vom Tisch, zeichnet sich vielmehr als Zukunftsszenario bereits deutlich $\mathrm{ab}$.

\section{III.}

Die hohen Krisenkosten, welche die breite Bevölkerung noch zu spüren bekommen wird, deuten auch auf ein fundamentales Problem des globalen Krisenmanagements hin. Viele strukturelle Probleme der Krise, etwa eine sinkende Lohnquote in den großen kapitalistischen Ländern, die zu immer weiteren Investitionen in den $\mathrm{Fi}$ nanzsektor beitrug, die flexiblen Wechselkurse und fehlenden Kapitalverkehrskontrollen oder die strukturellen Ungleichgewichte zwischen Überschuss- und Defizitländern, wurden im Zuge der öffentlichen Kriseninterventionspolitik nicht beseitigt. Die meisten von ihnen kehren in einem neuen Gewand wieder. So haben die Rettungsaktionen bereits zu einer erneuten Blasenbildung auf den Finanzmärkten geführt, der Dow Jones ist kürzlich das erste Mal seit der Krise wieder über die Marke von 10.000 geklettert. Auch die Vorstände der Großbanken lassen wieder die Korken knallen: Die Deutsche Bank hat ebenso wie die US-Großbanken Wells Fargo und Morgan Stanley im dritten Quartal 2009 Milliardengewinne eingefahren. Zugleich ist unklar, wie die immensen Krisenkosten 
langfristig bewältigt werden sollen. Das US-amerikanische Haushaltsdefizit von 1,4 Billionen US\$ weist darauf hin, dass bisher kein Weg gefunden wurde, die Schuldenpyramide zu verkleinern. Der frisch gebackene Finanzminister Schäuble rechnet mit einer Rekord-Neuverschuldung von 76 Mrd. Euro im Jahr 2010. Wird nach dem konjunkturpolitischen Strohfeuer perspektivisch wieder $\mathrm{zu}$ altbekannten Rezepten der Angebots- und Sparpolitik zurückgekehrt? Oder behalten ausgerechnet die neoklassischen Ökonomen Recht, dass den gewaltigen Konjunkturprogrammen schließlich eine massive Inflation folgt?

Alex Demirovic analysiert die staatliche Antikrisenpolitik in Deutschland und widerspricht der gängigen Meinung, dass der Staat zurückkehre und ein Ende des Neoliberalismus einläute: Der Staat war niemals weg und auch in der Krise werde die neoliberale Politik letzten Endes weitergeführt. Als „realistische“ linke Alternative zur staatlichen Krisenpolitik werden wieder verschiedene Varianten des Linkskeynesianismus propagiert. Mit Keynes und seinen heutigen Anhängern setzt sich Ingo Stützle kritisch auseinander. Zur Diskussion politischer Alternativen passt auch der außerhalb des Heftschwerpunkts liegende Beitrag von Silke van Dyk, bei dem es um die Frage geht, inwiefern der Kapitalismus fähig ist, Gegenstrategien in Ressourcen der eigenen Erneuerung zu verwandeln.

Es scheint gewiss, dass in vielen Industriestaaten kein Subjekt existiert, das fähig wäre, die Grundlagen für einen neuen „New Deal“ zu schaffen. Auch die Wende der US-Wirtschaftspolitik unter Franklin D. Roosevelt (1933-45) wurde erst durch eine starke Arbeiterbewegung ermöglicht. USPräsident Obama hat zwar direkt nach seinem Amtsantritt erkannt, dass es eines solchen reformpolitischen Subjekts bedürfte - die Gewerkschaften seien eben nicht Teil des Problems, sondern Teil der Lösung. Einstweilen aber dürften die Gewerkschaften, aufgrund ihres desolaten $\mathrm{Zu}$ stands nicht nur in den USA, als zentrale Akteure nationaler Krisenregulierung und -bewältigung ausscheiden. Begleitet wird dieses Problem von einem mindestens ebenso gewichtigen auf der Ebene der internationalen Politik: Die Vereinigten Staaten sind nicht mehr in der hegemonialen wirtschaftlichen Position, wie sie es einst unter Roosevelt waren. In den USA scheinen weder die Machtressourcen noch der politische Willen $\mathrm{zu}$ existieren, um ein zweites Bretton Woods-System zu stabilisieren. Es gibt in der Weltwirtschaft zwar erhebliche Machtverschiebungen wie von Dieter Boris und Stefan Schmalz in ihrem Beitrag diskutiert wird. Im aufstrebenden China gibt es zwar die wirtschaftlichen Mittel, um eine aktive Konjunkturpolitik vor Ort durchzusetzen, und offensichtlich auch keine ideologischen Barrieren für eine solche Politik. Aber auf der internationalen Ebene fehlt der chinesischen Regierung weiterhin der Einfluss, ihre Ideen etwa von einer neuen Weltwährungsarchitektur zu verwirklichen. Auch Indien kommt für eine gewichtige internationale Rolle noch längst nicht in Frage. Dafür sind nicht zuletzt die internen Probleme noch viel zu groß, wie dem Beitrag von Hanna Al Taher und Matthias Ebenau zu entnehmen ist. Die Strukturprobleme der Weltökonomie werden noch zunehmen, so dass der sich andeutende Aufschwung eher ein Zwischenaufschwung sein dürfte: Nach der Krise ist vor der Krise.

$$
* * *
$$

Am späten Abend des 29. September erlag Jürgen Hoffmann seinem langjährigen Krebsleiden. Jürgen war 1970 Mitbegründer der PROKLA und danach viele Jahre Redaktionsmitglied, einige Jahre davon geschäftsführender Redakteur. Auch nach seinem Ausscheiden aus der Redaktion blieb er der PROKLA als aktives Mitglied in der „Vereinigung der Kritik der politischen Ökonomie", von der die PROKLA herausgegeben wird, eng verbunden. Trotz Krankheit ließ sich Jürgen in den Redaktionsbeirat der PROKLA wählen und nahm bis zuletzt mit Hinweisen und Vorschlägen regen Anteil an der Redaktionsarbeit. Der PROKLA galt auch einer seiner letzten Akte: für seine Beerdigung wünschte er sich keine Blumen, sondern Spenden für die PROKLA. Rudi Schmidt würdigt Jürgen Hoffmann in einem Nachruf. Passend zum Heftschwerpunkt drucken wir aus Jürgens Buch „Politisches Handeln und gesellschaftliche Struktur" einen Text zur Weltwirtschaftskrise von 1929 ab. Jürgen wird uns fehlen. 